\title{
A Seven-Step Block Multistep Method for the Solution of First Order Stiff Differential Equations
}

\section{Solomon Gebregiorgis* and Hailu Muleta}

Department of Mathematics, Faculty of Natural Sciences, Jimma University, Ethiopia (*solomonggty@gmail.com).

\section{ABSTRACT}

In this paper, a seven-step block method for the solution of first order initial value problem in ordinary differential equations based on collocation of the differential equation and interpolation of the approximate solution using power series have been formed. The method is found to be consistent and zero-stable which guarantees convergence. Finally, numerical examples are presented to illustrate the accuracy and effectiveness of the method.

Keywords: Power series, Collocation, Interpolation, Block method, Stiff.

\section{INTRODUCTION}

This paper considers the general first order initial value problems of ordinary differential equations of the form:

$$
y^{\prime}(x)=f(x, y(x)), y\left(x_{0}\right)=y_{0}, x \in[a, b] .
$$

Equation (1) can be regarded as stiff if its exact solution contains very fast as well as very slow components (Dahlquist, 1974). Stiff IVPs occur in any fields of engineering and physical sciences. The solution is characterized by the presence of transient and steady state components, which restrict the step size of many numerical methods (Suleiman et al., 2015). This behavior makes it difficult to develop suitable methods for solving stiff problems. However, efforts have been made by researchers, such as Abasi et al. (2014), Alvarez and Rojo (2002), Cash (1980), Dahlquist (1974), Suleiman et al. (2015), Yatim et al. (2011), and Mohd Zawawi et al. (2015) among others, to develop methods for stiff ODEs.

Linear Multistep Method (LMM) is a computational procedure where by a numerical approximation $y_{n+j}$ to the exact solution $y\left(x_{n+j}\right)$ of the first order Initial Value Problems (IVPs) of equation (1) is obtained. In LMM to find the $k^{\text {th }}$ approximate value, we use the already calculated previous $k$ approximate values. Given a sequence of equally spaced mesh points $x_{n}$ with step size $h$, the general k-step LMM is as given in Lambert (1973) as:

$$
\sum_{j=0}^{k} \alpha_{j} y_{n+j}=h \sum_{j=0}^{k} \beta_{j} f_{n+j}
$$


where, the coefficients $\alpha_{1}, \alpha_{2}, \alpha_{3}, \ldots, \alpha_{k}$ and $\beta_{1}, \beta_{2}, \beta_{3}, \ldots, \beta_{k}$ are real constants.

It is shown that a power series method is effective in handling both linear as well as nonlinear problems (Hirayama, 2000). Abualanja (2015) used the power series method and developed a three-step continuous representation of a block implicit multistep scheme using interpolation of the approximate solution and collocation of derivative function with power series as basic functions for solving non-stiff first order ODEs. Most recently, Berhan et al. (2019) have modified the works of Abualanja (2015) to obtain a four-step block implicit multistep scheme for solving stiff first order ODEs. In this paper, we have extended the work of Berhan et al. (2019) to obtain a seven-step block scheme.

\section{DERIVATION OF THE PROPOSED METHOD}

To describe the method, we divide the interval $[a, b]$ into $N$ equal sub-intervals of mesh length $h$. Let $a=x_{0}, x_{1}, x_{2}, \cdots, x_{n}=b$ be the mesh points, then we have $x_{n}=x_{0}+n h, n=0(1) N$.Let the power series solutions of the equation (1) be $y(x)=\sum_{j=0}^{\infty} c_{j} x^{j}$.

The approximate solution of equation (1) will be:

$$
y(x)=\sum_{j=0}^{k} c_{j} x^{j} .
$$

Substituting equation (3) into equation (1), we get:

$$
y^{\prime}(x)=\sum_{j=0}^{k} j c_{j} x^{j-1} \approx f(x, y) \text {. }
$$

Now, by adding a perturbed term to equation (4), we obtain:

$$
\sum_{j=0}^{k} j c_{j} x^{j-1}=f(x, y)+\lambda L_{k}\left(x_{n+j}\right)
$$

where $\lambda$ is a perturbed parameter to be determined and $L_{k}\left(x_{n+j}\right)$ is the $k^{\text {th }}$ shifted Legendre polynomial obtained by the following recursive formula.

$$
L_{0}(x)=1, L_{1}(x)=x, \text { and }(n+1) L_{n+1}-(2 n+1) L_{n}+n L_{n-1}=0
$$

According to Suli and Mayers (2003), if a function is defined on $[a, b]$, it is sometimes necessary in the applications to expand the function in a series of orthogonal polynomials in this interval. Clearly the substitution: 


$$
x(t)=\frac{2}{b-a}\left[t-\frac{b+a}{2}\right], a<b .
$$

transforms the interval $[a, b]$ of the $t$-axis in to the interval $[-1,1]$ of the $x$-axis.

Note that the above equation is the same as:

$$
x(t)=\left[\frac{2 t-\left(x_{n+k}+x_{n}\right)}{x_{n+k}-x_{n}}\right], k=1,2,3, \cdots .
$$

with the substitution of $a=x_{n}$ and $b=x_{n+k}$. Here $x_{n+k}=x_{n}+k h$ where $h$ is the step size.

Using equation (7), $L_{k}\left(x_{n+j}\right)$ is transformed into $L_{k}(x(t))$ where $t=x_{n+j}, j=0(1) k$. From equation (5), we deduce that:

$$
c_{1}+2 x c_{2}+3 x^{2} c_{3}+\ldots+k x^{k-1} c_{k}=f(x, y)+\lambda L_{k}\left(x_{n+j}\right) .
$$

Interpolating equation (3) at $x=x_{n}$ and collocating equation (8) at $x_{n+j}, j=0,1,2, \ldots, k$ , we get a system of $k+2$ equations with $k+2$ unknowns.

$$
\begin{aligned}
& c_{0}+c_{1} x_{n}+c_{2} x_{n}^{2}+c_{3} x_{n}^{3}+\ldots+c_{k} x_{n}^{k}=y_{n} \\
& c_{1}+2 c_{2} x_{n}+3 c_{3} x_{n}^{2}+\ldots+k c_{k} x_{n}^{k-1}-\lambda L_{k}\left(x_{n}\right)=f_{n} \\
& c_{1}+2 c_{2} x_{n+1}+3 c_{3} x_{n+1}^{2}+\ldots+k c_{k} x_{n+1}^{k-1}-\lambda L_{k}\left(x_{n+1}\right)=f_{n+1}
\end{aligned}
$$

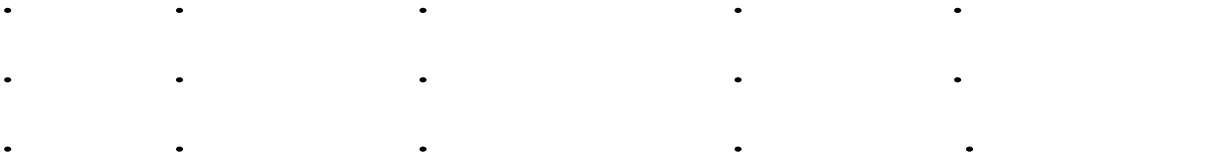

$$
\begin{aligned}
& c_{1}+2 c_{2} x_{n+k}+3 c_{3} x_{n+k}^{2}+\ldots+k c_{k} x_{n+k}^{k-1}-\lambda L_{k}\left(x_{n+k}\right)=f_{n+k}
\end{aligned}
$$

Solving equation (9), we get the values of the unknown parameters $\lambda, c_{0}, c_{1}, c_{2}, \ldots$, and $c_{k}$ .At this point, if we interpolate equation (3) at $x_{n+k}$, we get:

$$
y_{n+k}=c_{0}+c_{1} x_{n+k}+c_{2} x_{n+k}^{2}+\ldots+c_{k} x_{n+k}^{k} .
$$

The next task now becomes a matter of expressing equation (10) in terms of $y_{n}$ and $f_{n+k}, k=0(1) k$ after substituting the values of the unknown parameters. Maple software has been used to simplify such complicated task. 
Now in this study, we will derive the discrete schemes for $k=5,6$, and 7 . Please bear in mind that, in the ongoing discussions, we applied equality in $L_{k}\left(x_{n+j}\right)=L_{k}(x(t))$ where $t=x_{n+j}$ to mean just the left and right expressions are equal after transformation of $t=x_{n+j}$ to $x(t)=x\left(x_{n+j}\right)$.

\subsection{Derivation of the Method for $k=5$}

Using equation (6) the Legendre polynomial becomes:

$$
\begin{gathered}
L_{5}(x)=\frac{1}{8}\left(15 x-70 x^{3}+63 x^{5}\right) \text { and applying equation (7), we get: } \\
L_{5}\left(x_{n}\right)=L_{5}(-1)=-1, L_{5}\left(x_{n+1}\right)=L_{5}\left(-\frac{3}{5}\right)=\frac{447}{3125}, L_{5}\left(x_{n+2}\right)=L_{5}\left(-\frac{1}{5}\right)=-\frac{961}{3125}, \\
L_{5}\left(x_{n+3}\right)=L_{5}\left(\frac{1}{5}\right)=\frac{961}{3125}, L_{5}\left(x_{n+4}\right)=L_{5}\left(\frac{3}{5}\right)=-\frac{447}{3125}, \text { and } L_{5}\left(x_{n+5}\right)=L_{5}(1)=1
\end{gathered}
$$

Now equation (8) becomes:

$$
\begin{aligned}
& c_{0}+c_{1} x_{n}+c_{2} x_{n}^{2}+c_{3} x_{n}^{3}+c_{4} x_{n}^{4}+c_{5} x_{n}^{5}=y_{n} \\
& c_{1}+2 c_{2} x_{n}+3 c_{3} x_{n}^{2}+4 c_{4} x_{n}^{3}+5 c_{5} x_{n}^{4}-\lambda=f_{n} \\
& c_{1}+2 c_{2} x_{n+1}+3 c_{3} x_{n+1}^{2}+4 c_{4} x_{n+1}^{3}+5 c_{5} x_{n+1}^{4}+\frac{447}{3125} \lambda=f_{n+1} \\
& c_{1}+2 c_{2} x_{n+2}+3 c_{3} x_{n+2}^{2}+4 c_{4} x_{n+2}^{3}+5 c_{5} x_{n+2}^{4}-\frac{961}{3125} \lambda=f_{n+2} \\
& c_{1}+2 c_{2} x_{n+3}+3 c_{3} x_{n+3}^{2}+4 c_{4} x_{n+3}^{3}+5 c_{5} x_{n+3}^{4}+\frac{961}{3125} \lambda=f_{n+3} \\
& c_{1}+2 c_{2} x_{n+4}+3 c_{3} x_{n+4}^{2}+4 c_{4} x_{n+4}^{3}+5 c_{5} x_{n+4}^{4}-\frac{447}{3125} \lambda=f_{n+4} \\
& c_{1}+2 c_{2} x_{n+5}+3 c_{3} x_{n+5}^{2}+4 c_{4} x_{n+5}^{3}+5 c_{5} x_{n+5}^{4}+\lambda=f_{n+5}
\end{aligned}
$$

The resulting system of equations, equation (11) is solved using Maple software for $c_{0}, c_{1}, c_{2}, c_{3}, c_{4}, c_{5}$, and $\lambda$ and then substituted in equation (10) to get:

$$
y_{n+5}=y_{n}+\frac{h}{288}\left(95 f_{n}+375 f_{n+1}+250 f_{n+2}+250 f_{n+3}+375 f_{n+4}+95 f_{n+5}\right) .
$$

Therefore, equation (12) is the numerical scheme when $k=5$.

\subsection{Derivation of the Method for $k=6$}

Using equation (6) the Legendre polynomial for $k=6$ becomes: 


$$
\begin{aligned}
& L_{6}(x)=\frac{1}{16}\left(-5+105 x^{2}-315 x^{4}+231 x^{6}\right) \text { and applying equation }(7), \text { we get: } \\
& L_{6}\left(x_{n}\right)=L_{6}(-1)=1, L_{6}\left(x_{n+1}\right)=L_{5}\left(-\frac{2}{3}\right)=-\frac{67}{3888}, L_{6}\left(x_{n+2}\right)=L_{6}\left(-\frac{1}{3}\right)=-\frac{47}{243}, \\
& L_{6}\left(x_{n+3}\right)=L_{5}(0)=\frac{961}{3125}, L_{6}\left(x_{n+4}\right)=L_{5}\left(\frac{1}{3}\right)=\frac{47}{243}, L_{6}\left(x_{n+5}\right)=L_{5}\left(\frac{2}{3}\right)=-\frac{67}{3888}, \text { and } \\
& L_{6}\left(x_{n+6}\right)=L_{6}(1)=1 .
\end{aligned}
$$

Now equation (8) becomes:

$$
\begin{aligned}
& c_{0}+c_{1} x_{n}+c_{2} x_{n}^{2}+c_{3} x_{n}^{3}+c_{4} x_{n}^{4}+c_{5} x_{n}^{5}+c_{6} x_{n}^{6}=y_{n} \\
& c_{1}+2 c_{2} x_{n}+3 c_{3} x_{n}^{2}+4 c_{4} x_{n}^{3}+5 c_{5} x_{n}^{4}+6 c_{6} x_{n}^{5}+\lambda=f_{n} \\
& c_{1}+2 c_{2} x_{n+1}+3 c_{3} x_{n+1}^{2}+4 c_{4} x_{n+1}^{3}+5 c_{5} x_{n+1}^{4}+6 c_{6} x_{n+1}^{5}-\frac{67}{3888} \lambda=f_{n+1} \\
& c_{1}+2 c_{2} x_{n+2}+3 c_{3} x_{n+2}^{2}+4 c_{4} x_{n+2}^{3}+5 c_{5} x_{n+2}^{4}+6 c_{6} x_{n+2}^{5}+\frac{47}{243} \lambda=f_{n+2} \\
& c_{1}+2 c_{2} x_{n+3}+3 c_{3} x_{n+3}^{2}+4 c_{4} x_{n+3}^{3}+5 c_{5} x_{n+3}^{4}+6 c_{6} x_{n+3}^{5}-\frac{5}{6} \lambda=f_{n+3} \\
& c_{1}+2 c_{2} x_{n+4}+3 c_{3} x_{n+4}^{2}+4 c_{4} x_{n+4}^{3}+5 c_{5} x_{n+4}^{4}+6 c_{6} x_{n+4}^{5}+\frac{47}{243} \lambda=f_{n+4} \\
& c_{1}+2 c_{2} x_{n+5}+3 c_{3} x_{n+5}^{2}+4 c_{4} x_{n+5}^{3}+5 c_{5} x_{n+5}^{4}+6 c_{6} x_{n+5}^{5}-\frac{67}{3888} \lambda=f_{n+5} \\
& c_{1}+2 c_{2} x_{n+6}+3 c_{3} x_{n+6}^{2}+4 c_{4} x_{n+6}^{3}+5 c_{5} x_{n+6}^{4}+6 c_{6} x_{n+6}^{5}+\lambda=f_{n+6}
\end{aligned}
$$

The resulting system of equations, equation (13) is solved for $c_{0}, c_{1}, c_{2}, c_{3}, c_{4}, c_{5}, \mathrm{c}_{6}$, and $\lambda$ and substituted into equation (10) to get:

$$
y_{n+6}=y_{n}+\frac{h}{140}\left(41 f_{n}+216 f_{n+1}+27 f_{n+2}+272 f_{n+3}+27 f_{n+4}+216 f_{n+5}+41 f_{n+6}\right) .
$$

Therefore, equation (14) is the implicit scheme for $k=6$.

\subsection{Derivation of the Method for $k=7$}

In a similar procedure as in the previous sections, we obtain an implicit scheme for $k=7$ as follows:

$$
\begin{array}{r}
y_{n+7}=y_{n}+\frac{h}{17280}\left(5257 f_{n}+25039 f_{n+1}+9261 f_{n+2}+20923 f_{n+3}+\right. \\
\left.20923 f_{n+4}+9261 f_{n+5}+25039 f_{n+6}+5257 f_{n+7}\right)
\end{array}
$$




\subsection{The Proposed Block Method}

The proposed block procedure with implicit linear multistep method is as follows.

$$
\begin{aligned}
y_{n+1}= & y_{n}+\frac{h}{2}\left(f_{n}+f_{n+1}\right) \\
y_{n+2}= & y_{n}+\frac{h}{3}\left(f_{n}+4 f_{n+1}+f_{n+2}\right) \\
y_{n+3}= & y_{n}+\frac{h}{8}\left(3 f_{n}+9 f_{n+1}+9 f_{n+2}+3 f_{n+3}\right) \\
y_{n+4}= & y_{n}+\frac{h}{45}\left(14 f_{n}+64 f_{n+1}+24 f_{n+2}+64 f_{n+3}+14 f_{n+4}\right) \\
y_{n+5}= & y_{n}+\frac{h}{288}\left(95 f_{n}+375 f_{n+1}+250 f_{n+2}+250 f_{n+3}+375 f_{n+4}+\right. \\
& \left.95 f_{n+5}\right) \\
y_{n+6}= & y_{n}+\frac{h}{140}\left(41 f_{n}+216 f_{n+1}+27 f_{n+2}+272 f_{n+3}+27 f_{n+4}+\right. \\
& \left.216 f_{n+5}+41 f_{n+6}\right) \\
y_{n+7} & y_{n}+\frac{h}{17280}\left(5257 f_{n}+25039 f_{n+1}+9261 f_{n+2}+20923 f_{n+3}+\right. \\
& \left.20923 f_{n+4}+9261 f_{n+5}+25039 f_{n+6}+5257 f_{n+7}\right)
\end{aligned}
$$

\section{ANALYSIS OF THE METHOD}

\subsection{Order and Error Constant}

For a given linear multistep method, the so called characteristic polynomials are defined as:

$$
\rho(z)=\sum_{j=0}^{k} \alpha_{j} z^{j} \text { and } \sigma(z)=\sum_{j=0}^{k} \beta_{j} z^{j}
$$

According to Lambert (1973) and Suli and Mayers (2003), the local truncation error associated with equation (2) is defined by the difference operator:

$$
L[y(x): h]=\frac{1}{h \sum_{j=0}^{k} \beta_{j}}\left(\sum_{j=0}^{k}\left[\alpha_{j} y\left(x_{n}+j h\right)-h \beta_{j} f\left(x_{n}+j h\right)\right]\right)
$$

where, $y(x)$ is the exact solution.

Suppose $y(x)$ is smooth and expanding equation (17) and applying Taylor expansion on $y\left(x_{n}+j h\right)$ and $f\left(x_{n}+j h\right)$ yields: 


$$
L[y(x): h]=\frac{1}{h \sigma(1)}\left[c_{0} y\left(x_{n}\right)+c_{1} h y^{\prime}\left(x_{n}\right)+c_{2} h^{2} y^{\prime \prime}\left(x_{n}\right)+\ldots+c_{p+1} h^{p+1} y^{p+1}\left(x_{n}\right)\right]
$$

and the coefficients are as follows.

$$
c_{0}=\sum_{j=0}^{k} \alpha_{j}, c_{1}=\sum_{j=1}^{k} j \alpha_{j}-\sum_{j=0}^{k} \beta_{j}, c_{2}=\sum_{j=1}^{k} \frac{j^{2}}{2 !} \alpha_{j}-\sum_{j=1}^{k} j \beta_{j}, c_{p}=\sum_{j=1}^{k} \frac{j^{p}}{p !} \alpha_{j}-\sum_{j=1}^{k} \frac{j^{p-1}}{(p-1) !} \beta_{j}
$$

A given LMM is of order $p$ if all the coefficients $\left(c_{i}{ }^{\prime} s\right)$ are zero except the last coefficient $\left(c_{p+1}\right)$. Moreover, the number $\frac{c_{p+1}}{\sigma(1)}$ is called the error constant of the method. Hence, the orders of the methods in equation (16) are $\left(\begin{array}{lllllll}2 & 4 & 4 & 6 & 6 & 8 & 8\end{array}\right)^{T}$ with error constants $\left(-\frac{1}{12}-\frac{1}{180}-\frac{1}{80}-\frac{8}{945}-\frac{55}{12096}-\frac{3}{2800}-\frac{68}{30155}\right)^{T}$

\subsection{Zero Stability of the Method}

Applying the works of Shampine and Watts (1969), equation (16) is expressed as a block formula in matrix form:

$$
A Y_{M}=E y_{n}+h d f\left(y_{n}\right)+h b F\left(Y_{M}\right)
$$

Where,

$$
\begin{aligned}
& A Y_{M}=\left[\begin{array}{lllllll}
1 & 0 & 0 & 0 & 0 & 0 & 0 \\
0 & 1 & 0 & 0 & 0 & 0 & 0 \\
0 & 0 & 1 & 0 & 0 & 0 & 0 \\
0 & 0 & 0 & 1 & 0 & 0 & 0 \\
0 & 0 & 0 & 0 & 1 & 0 & 0 \\
0 & 0 & 0 & 0 & 0 & 1 & 0 \\
0 & 0 & 0 & 0 & 0 & 0 & 1
\end{array}\right]\left[\begin{array}{c}
y_{n+1} \\
y_{n+2} \\
y_{n+3} \\
y_{n+4} \\
y_{n+5} \\
y_{n+6} \\
y_{n+7}
\end{array}\right], E Y_{n}=\left[\begin{array}{lllllll}
0 & 0 & 0 & 0 & 0 & 0 & 1 \\
0 & 0 & 0 & 0 & 0 & 0 & 1 \\
0 & 0 & 0 & 0 & 0 & 0 & 1 \\
0 & 0 & 0 & 0 & 0 & 0 & 1 \\
0 & 0 & 0 & 0 & 0 & 0 & 1 \\
0 & 0 & 0 & 0 & 0 & 0 & 1 \\
0 & 0 & 0 & 0 & 0 & 0 & 1
\end{array}\right]\left[\begin{array}{c}
y_{n-6} \\
y_{n-5} \\
y_{n-4} \\
y_{n-3} \\
y_{n-2} \\
y_{n-1} \\
y_{n}
\end{array}\right], \\
& d f\left(y_{n}\right)=\left[\begin{array}{ccccccc}
0 & 0 & 0 & 0 & 0 & 0 & \frac{1}{2} \\
0 & 0 & 0 & 0 & 0 & 0 & \frac{1}{3} \\
0 & 0 & 0 & 0 & 0 & 0 & \frac{3}{8} \\
0 & 0 & 0 & 0 & 0 & 0 & \frac{14}{45} \\
0 & 0 & 0 & 0 & 0 & 0 & \frac{95}{288} \\
0 & 0 & 0 & 0 & 0 & 0 & \frac{41}{140} \\
0 & 0 & 0 & 0 & 0 & 0 & \frac{5257}{17280}
\end{array}\right]\left[\begin{array}{l}
f_{n-6} \\
f_{n-3} \\
f_{n-4} \\
f_{n-3} \\
f_{n-2} \\
f_{n-1} \\
f_{n}
\end{array}\right], \text { and }
\end{aligned}
$$




$$
b f\left(Y_{M}\right)=\left[\begin{array}{llllllll}
\frac{1}{2} & 0 & 0 & 0 & 0 & 0 & 0 \\
\frac{4}{3} & \frac{1}{3} & 0 & 0 & 0 & 0 & 0 \\
\frac{9}{8} & \frac{9}{8} & \frac{3}{8} & 0 & 0 & 0 & 0 & 0
\end{array}\right]\left[\begin{array}{l}
f_{n+1} \\
f_{n+2} \\
f_{n+3} \\
f_{n+4} \\
f_{n+5} \\
f_{n+6} \\
f_{n+7}
\end{array}\right]
$$

The stability polynomial for equation (16) is obtained by evaluating

$$
\operatorname{det}[A z-E]=0 \text {. }
$$

to get the characteristic polynomial as follows:

$$
z^{7}-z^{6}=0
$$

Solving equation (22) for $z$ gives the following roots:

$$
z_{1}=0, z_{2}=0, z_{3}=0, z_{4}=0, z_{5}=0, z_{6}=0 \text {, and } z_{7}=1 \text {. }
$$

Owing to the work of Fatunla (1988), Our block method equations are zero stable since $\left|z_{j}\right| \leq 1, j=1,2,3,4,5,6$, and 7 and for those roots with $\left|z_{j}\right|=1$, the multiplicity does not exceed two.

\subsection{Consistency of the Method}

Referring to the definition given by Lambert (1973) which states that a LMM is said to be consistent if it has order at least one, the block scheme given by equation (16) is consistent.

\subsection{Convergence of the Method}

Owing to Dahlquist theorem which states that the necessary and sufficient condition for a LMM to be convergent is to be consistent and zero stable, it is clear that the method given by equation (16) is convergent as it is both consistent and zero stable.

\section{NUMERICAL EXAMPLES}

For the purpose of showing the effectiveness of our method, comparisons are made with previous related works using the following stiff first order ODEs as follows. 
Example 1: Consider the first order stiff ordinary differential equation, Suleiman et al. (2015).

$$
y^{\prime}(x)=\frac{y(1-y)}{2 y-1} \quad y(0)=\frac{5}{6} \quad 0 \leq x \leq 1 .
$$

The exact solution is $y(x)=\frac{1}{2}+\sqrt{\frac{1}{4}-\frac{5}{36} e^{-x}}$.

Example 2. Consider the first order stiff ordinary differential equation, Ibrahim (2006).

$$
y^{\prime}(x)=-20 y+20 \sin x+\cos x \quad y(0)=1 \quad x \in[0,2] .
$$

The exact solution is $y(x)=\sin x+e^{-20 x}$.

Table 1. Maximum Absolute errors (MAEs) of Suleiman et al. (2015), Yoseph Berhan (2017) and the Present Method (PM) for problem 1.

\begin{tabular}{|lccc|}
\hline $\boldsymbol{h}$ & $\begin{array}{c}\text { Suleiman et al. } \\
\text { (2015) }\end{array}$ & $\begin{array}{c}\text { Yoseph Berhan (2017) } \\
\text { (Block Method k1 up to k4) }\end{array}$ & $\begin{array}{c}\text { PM } \\
\text { (Block Method k1 up to } k 7)\end{array}$ \\
\hline $10^{-1}$ & - & $1.34333 \mathrm{e}-4$ & $5.63131 \mathrm{e}-5$ \\
\hline $10^{-2}$ & $1.47080 \mathrm{e}-3$ & $1.17710 \mathrm{e}-6$ & $6.83365 \mathrm{e}-8$ \\
\hline $10^{-3}$ & $1.52651 \mathrm{e}-4$ & $1.17799 \mathrm{e}-8$ & $7.00620 \mathrm{e}-11$ \\
\hline $10^{-4}$ & $1.53220 \mathrm{e}-5$ & $1.17705 \mathrm{e}-10$ & $7.03881 \mathrm{e}-14$ \\
\hline $10^{-5}$ & $7.10611 \mathrm{e}-8$ & $1.42827 \mathrm{e}-14$ & $7.24374 \mathrm{e}-19$ \\
\hline
\end{tabular}

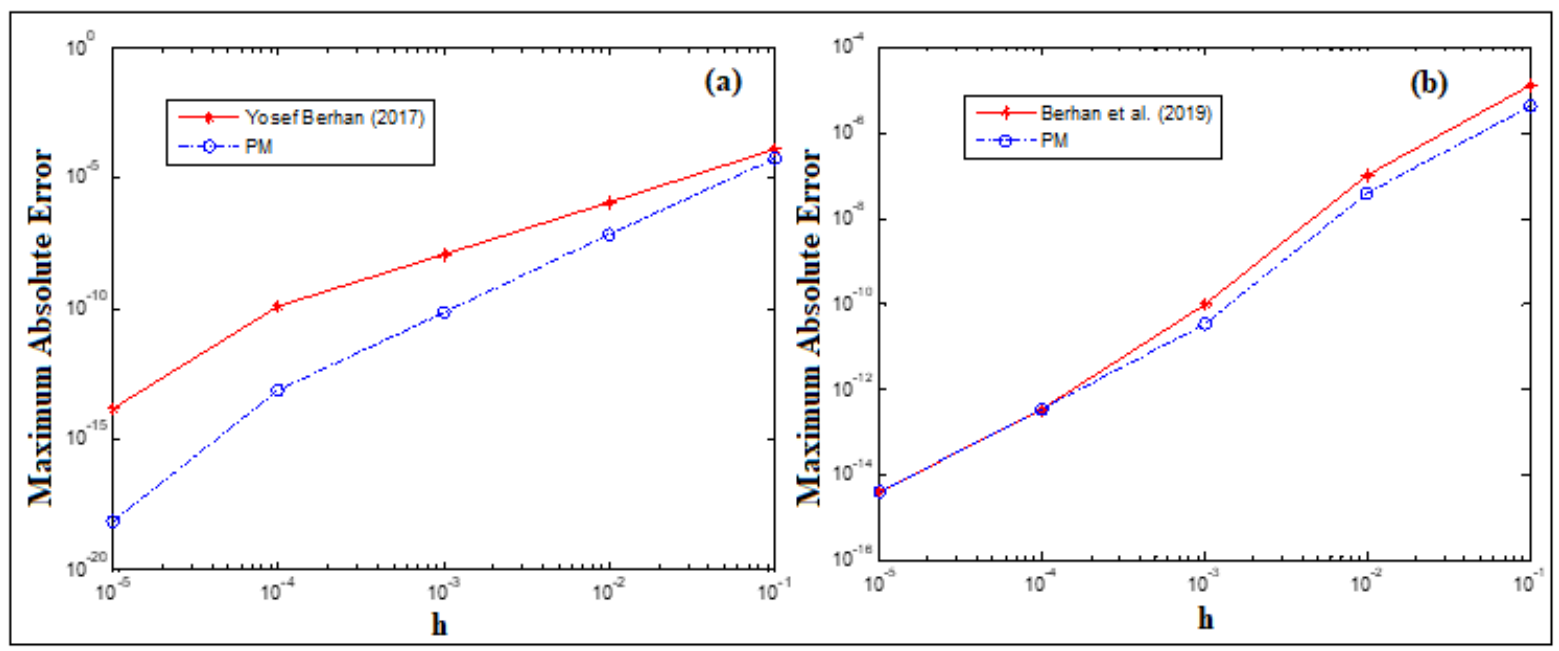

Figure 1. Log- $\log$ plot of the absolute maximum errors for (a) problem 1 and (b) problem 2.

\section{CONCLUSION}

In this study a block procedure with implicit eighth order linear multistep method using the power series as a basis function and by adding a perturbed term using Legendre polynomials is derived for the solutions of stiff first order differential equations. This method is based on 
collocation of the differential equation and interpolation of the approximate solution of power series at the grid points which is built on the discrete steps $\mathrm{k}=1,2,3$, and 4 .

The method is tested and found to be consistent, zero stable and hence convergent. We tested the method on two numerical examples and the numerical results depict that the method is accurate and effective for stiff problems. The results showed that our method's accuracy results are superior to that of the works of Berhan et al. (2019).

Finally, we recommend that further researches have to be done to optimize our method by using a different basis function and/or a perturbed term.

\section{ACKNOWLEDGEMENTS}

The authors would like to thank the anonymous reviewers for their valuable comments, suggestion and ideas that have improved the quality of the paper significantly.

\section{CONFLICT OF INTERESTS}

There are no conflicts of interest.

\section{REFERENCE}

Abasi, N., Suleiman M., Abbasi, N \& Musa, H. 2014. 2-point block BDF method with off-step points for solving stiff ODEs. Journal of Soft Computing and Applications, Volume 2014: 15p (DOI: 10.5899/2014/jsca-00039).

Abualnaja, K. 2015. A block procedure with LMMs using Legendre polynomials for Solving ODEs. Applied mathematics series, 6(1): 717-723.

Alvarez, J \& Rojo, J. 2002. An improved class of generalized Runge-Kutta methods for stiff problems. Part I: The scalar case. Appl. Math. Computer, 130: 537-560.

Berhan, Y., Gofe, G \& Gebregiorgis, S. 2019. Block procedure with implicit sixth order linear multistep method using legendre polynomials for solving stiff initial value problems. J. Fundam. Appl. Sci., 11(1): 1-10.

Cash, J. R. 1980. On the integration of stiff systems of ODEs using extended backward differentiation formulae. Numer. Math., 34: 235-246.

Dahlquist, G. 1974. Problems related to the numerical treatment of stiff differential equations. In: Gunther et al (eds.), International Computing Symposium, 1973, North Holland, Amsterdam, pp 307-314. 
Fatunla, S. 1988. Numerical Methods for initial value problems for ordinary differential equations. $1^{\text {st }}$ edition, eBook ISBN: 9781483269269, Academy Press, 308p.

Hirayama, H. 2000. Arbitrary Order and A-Stable Numerical Method for Solving Algebraic Ordinary Differential Equation by Power Series. 2nd International Conference on Mathematics and Computers in Physics, Vouliagmeni, Athens, 9-16 July 2000, 1-6.

Ibrahim, Z.B. 2006. Block multistep methods for solving ordinary differential equations, $\mathrm{PhD}$ Thesis, Universiti Putra Malysia.

Lambert, J.D. 1973. Computational methods in ordinary differential equations.John Willey and Sons, 278p. .

Mohd Zawawi, I, S., Ibrahim, Z. B \& Othman, K. I. 2015. Derivation of diagonally implicit block backward differentiation formulas for solving stiff initial value problems. Article ID 179231 Mathematical Problems in Engineering, 19p (DOI: 10.1155/2015/179231).

Shampine, L \& Watts, H. 1969. Block implicit one-step methods. Journal of Computer Maths., 23: 731-740 (http://dx.doi.org/10.1090/S0025-5718-1969-0264854-5).

Suleiman, M.B., Musa, M \& Ismail, F., 2015. An Implicit 2-point Block Extended Backward Differentiation Formula for Integration of Stiff Initial Value Problems. Malaysian Journal of Mathematical Science, 9(1): 33-51.

Süli, E \& Mayers, D. F. 2003. An introduction to numerical analysis. Cambridge University Press (DOI: 10.1017/CBO9780511801181).

Yatim, S. A. M., Ibrahim, Z. B., Othman, K. I \& Suleiman, M. B. 2011. A quantitative comparison of numerical method for solving stiff ordinary differential equations. Article ID 193691, Mathematical Problems in Engineering, 12p (DOI: $10.1155 / 2011 / 193691)$.

Yoseph B., Genanew, G \& Solomon, G. 2017. Block procedure with implicit sixth order linear multistep method using legendre polynomials for solving stiff initial value problems. MSc Thesis (Unpubl.). 